\title{
Luxaciones y fracturas luxaciones perilunares del carpo. Revisión de nuestra casuística
}

\author{
R. Franco Gómez ${ }^{(1)}$, M. Bosch Aguilía ${ }^{(2)}$, I. López Moreno ${ }^{(3)}$ \\ Hospital Asepeyo Sant Cugat, Sant Cugat del Vallés, Barcelona \\ (I) MÉdico RESIDENTE DE CIRUGÍA ORTOPÉDICA Y TRAUMATOLOGÍA

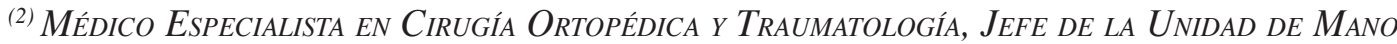

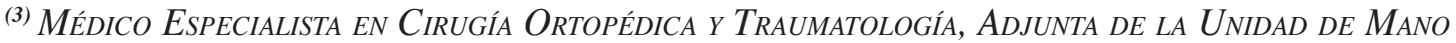

Correspondencia:

Dr. Raúl Franco Gómez

Avenida Alcalde Barnils, 54-60

08174, Sant Cugat del Vallés, Barcelona

Teléfono: 935653900

Fax: 935896368

e-mail: raulfrancog@gmail.com

Introducción: Las fracturas luxaciones perilunares del carpo son lesiones graves y poco frecuentes, producto de mecanismos de alta energía y que suelen asociarse a lesiones en otras localizaciones. Revisamos nuestra casuística de esta lesión valorando los resultados clínicos.

Material y Método: Es una serie de 33 casos identificados al valorar retrospectivamente las historias clínicas de los pacientes atendidos en urgencias entre enero de 1987 a junio de 2007.

Resultados: los 33 casos tuvieron un seguimiento medio de 14 meses. La valoración del resultado clínico se realizó en 21 pacientes, siendo en la mayoría pobre o malo, se encontró diferencia en el resultado clínico según la clasificación de la lesión y el tratamiento realizado.

Conclusiones: Es una lesión grave y poco frecuente que recibe un tratamiento heterogéneo. Probablemente el pronóstico se relacione con el tipo de tratamiento que reciben los pacientes y la clasificación de Conney. Debido a la escasa incidencia de esta patología es necesario protocolizar el tratamiento de los pacientes que presentan estas lesiones para obtener los mejores resultados posibles en cada caso.

Palabras clave: luxación carpo, fractura-luxación semilunar, perilunar
Introduction: Carpus perilunate dislocation and fracture dislocation are serious lesions, with low frequency, being produce by a high energy mechanism and usually associated to lesions in other locations. Material and Methods: Retrospective study of 33 patients assisted at Emergency Room since January 1987 to June 2007.

Results: The clinical evaluation was done in 21 patients, with poor results in most of them. Different results were obtained on the classification of the lesion and the treatment done.

Conclusions: It is a serious lesion treated in different and heterogeneous ways and the prognostic could depend in the type of lesion according to Cooney's classification and to the type of treatment. Due its low incidence it is necessary a protocol of treatment.

Key words: carpus dislocation, fracture-dislocation semilunate, perilunate. 


\section{INTRODUCCIÓN}

T

a muñeca tiene la función de proveer de movimiento a la mano y transmitir la fuerza desde el antebrazo, debiendo realizar esta tarea con la máxima estabilidad y movilidad. Para ello se configura en una compleja geometría, sin inserciones musculares en la primera hilara del carpo, dependiendo su estabilidad de la acción de ligamentos extrínsecos e intrínsecos repartidos en la vertiente dorsal y volar. Entre los huesos destaca la importancia del semilunar que soporta la mayor cantidad de la fuerza axial y el escafoides que sirve de nexo entre la hilera proximal y distal ${ }^{1}$.

Estudiando el mecanismo de esta lesión, en cadáveres, Mayfield et al..$^{2,3}$ concluyeron que se producía por un mecanismo de hiperextensión, desviación cubital y supinación intercapiana; como el movimiento que se hace al caer sobre la palma de la mano mientras se prona el antebrazo. Además describió una serie de lesiones ligamentosas que se producen de manera secuencial según aumentaba la energía del traumatismo, acuñando el término inestabilidad perilunar progresiva en la cual distinguió 4 estadios.

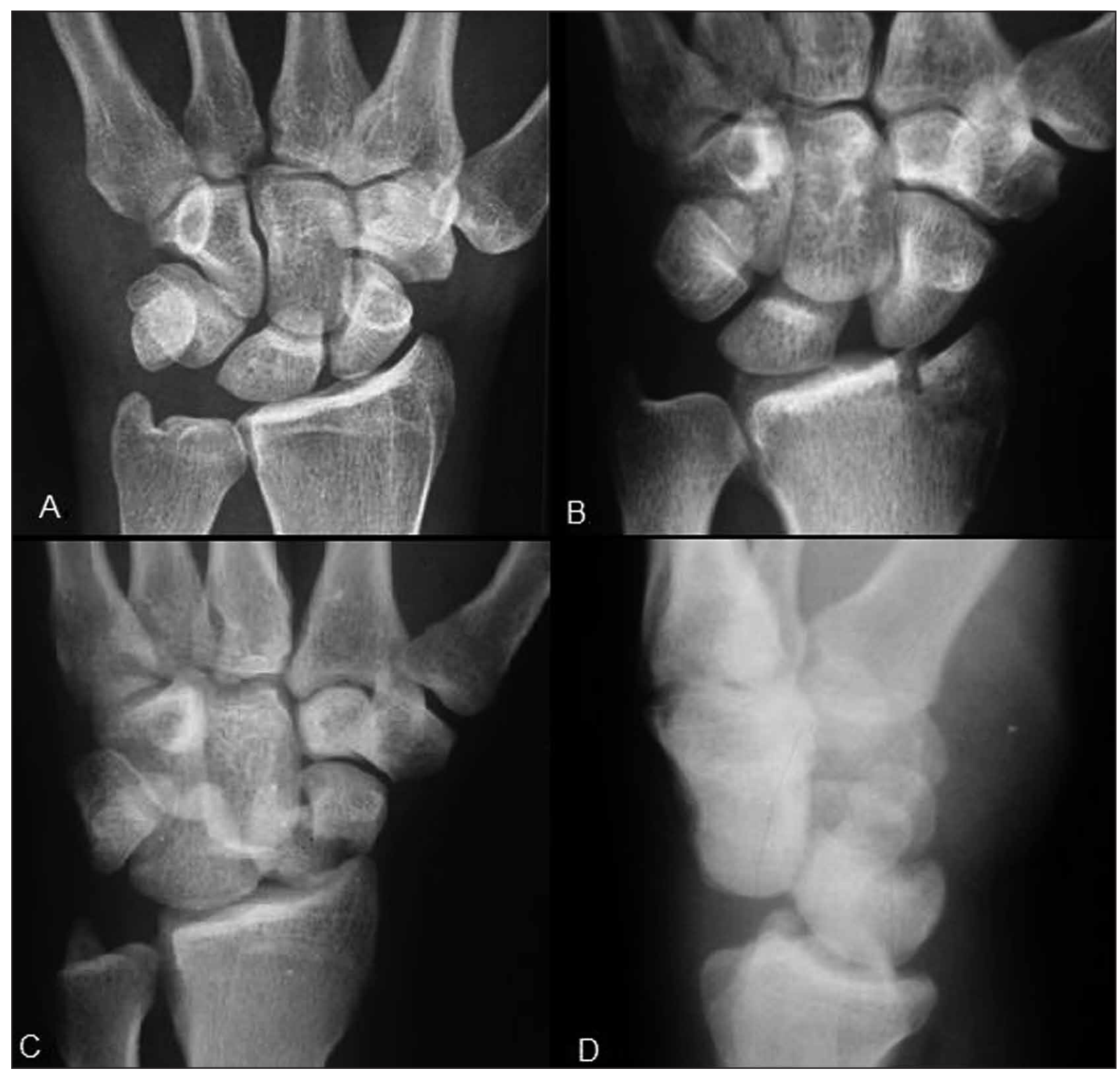

Figura 1. Hallazgos radiológicos. A. Radiografía antero-posterior de carpo normal; B. Aumento de espacio escafosemilunar; C. Disrupción de las líneas articulares del carpo; D. luxación dorsal del hueso grande. 


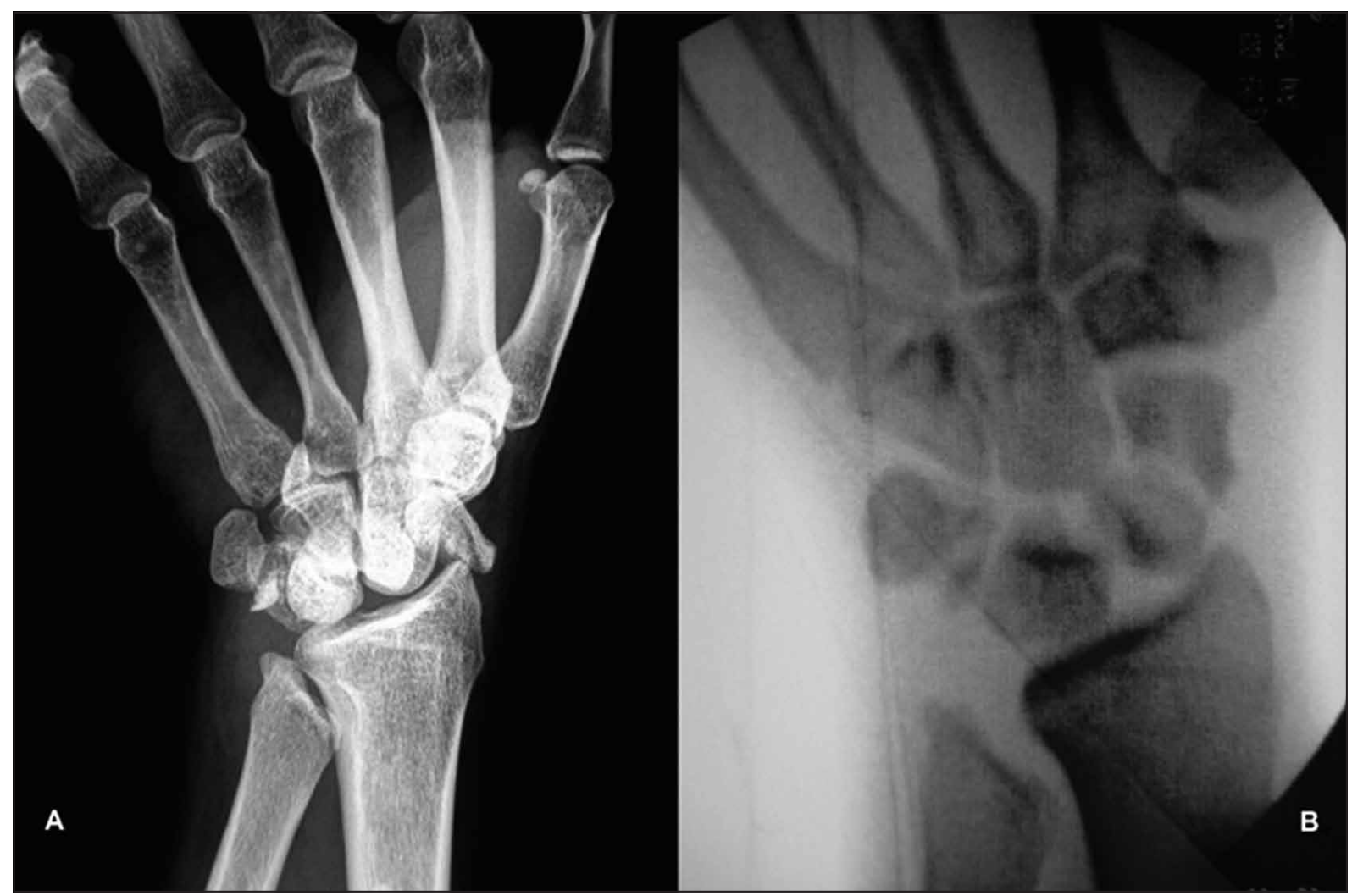

Figura 2. Ejemplo de valoración dinámica en tracción. A. Radiografía AP inicial. B. imagen obtenida durante la valoración dinámica en tracción en la que se evidencia fractura de escafoides y del piramidal.

El diagnóstico de urgencia debe orientarse con la exploración física y radiológica. De esta forma, en la región de la muñeca, se observará edema, deformidad, limitación para la movilización y dolor a la palpación. Debido a la probabilidad de lesión neurológica es necesario consignar el estado neurológico de la mano. En una adecuada radiología simple, que debe ser comparativa, podremos sospechar esta lesión si en la proyección antero-posterior, al valorar los arcos de Gilula, es imposible delinear sin disrupciones o intersecciones la superficie articular proximal y distal de la primera hilera y proximal de la segunda hilera ${ }^{4}$ o encontramos el aumento asimétrico del espacio escafo-semilunar (signo de Terry Thomas), también se podría observar una imagen compuesta de aspecto circular en el polo distal del escafoides (signo del anillo) aunque este último es un hallazgo común en muñecas sanas ${ }^{5}$. En la proyección lateral se deberá confirmar la adecuada congruencia articular entre el radio y el semilunar y de éste con el hueso grande; también podremos valorar el ángulo escafo-semilunar que debe estar entre $30^{\circ}$ y $60^{\circ}$ (Figura 1).

La tomografía axial computarizada puede utilizarse para identificar las lesiones óseas asociadas y valorar el grado de conminución. Más asequible en urgencias, es la posibilidad de realizar una exploración radiológica bajo tracción, previa anestesia local, que permitirá observar fracturas y sospechar lesiones ligamentosas ${ }^{6}$ ocultas a las radiografías simples, así como un intento de reducción cerrada (Figura 2).

Para clasificar estas lesiones existen varios métodos $^{7,8}$, sin que se haya demostrado alguna ventaja entre ellos para realizar una decisión terapéutica o un pronóstico. Del mismo modo tampoco se ha identificado una indicación terapéutica ideal. Los objetivos del tratamiento son la reducción anatómica, el mínimo periodo de inmovilización y el tratamiento rehabilitador adecuado a la lesión del paciente ${ }^{9}$. Para conseguirlos se deben tener en cuenta dos metas: primera restaurar la alineación entre el radio, el semilunar y el hueso grande; y segunda reparar 
las estructuras lesionadas que han permitido la luxación ${ }^{6}$.

Generalmente es posible la reducción de manera cerrada, cuando no han trascurrido más de 24 horas de la lesión y las fracturas no están desplazadas, vigilando atentamente la correcta alineación del escafoides que tiende a la rotación. Sin embargo, aunque se haya obtenido una adecuada reducción de manera cerrada se recomienda realizar una fijación ósea, ya que se ha observado que, hasta dos tercios de los pacientes pierden la reducción si esta se contiene únicamente por una escayola ${ }^{8}$. Para la fijación ósea suele ser suficiente la utilización de agujas de Kirschner generalmente de $1,5 \mathrm{~mm}$, pasando una del escafoides al semilunar y otra del escafoides al hueso grande. En los casos de fractura-luxación se añadirá a esta fijación la osteosíntesis propia de la fractura que acompañe a la luxación. Conforme pasan los días se hace poco probable la reducción cerrada debido a la interposición de partes blandas y la fibrosis. Por eso, pasado este tiempo, es preferible la osteosíntesis abierta y reparación o plastia ligamentosa según corresponda (Figura 3).

En la mayoría de las series el resultado clínico de estas lesiones es malo o pobre, evolucionando hacia la artrosis postraumática en el 56\% de los casos. Entre los factores que se han relacionado con un peor pronóstico se encuentran las lesiones abiertas y aquellas que fueron tratadas después de una semana de su producción ${ }^{7,10}$.

El objetivo de este trabajo es revisar la epidemiología de estas lesiones y el resultado del tratamiento realizado, para estandarizar el diagnóstico y tratamiento de las fractura-luxaciones perilunares del carpo.

\section{MATERIAL Y MÉTODO}

En 2007 se inició la revisión de historias clínicas de los pacientes que fueron diagnosticados en el Instituto Anglí de Barcelona y posteriormente en el Hospital Asepeyo Sant Cugat con esta patología, durante el periodo 1987-2007, encontrando 33 casos entre las 130.284 visitas que se realizaron en los servicios de urgencias de dichos centros. En estos 33 casos se realizó el estudio epidemiológico de la lesión. Aunque

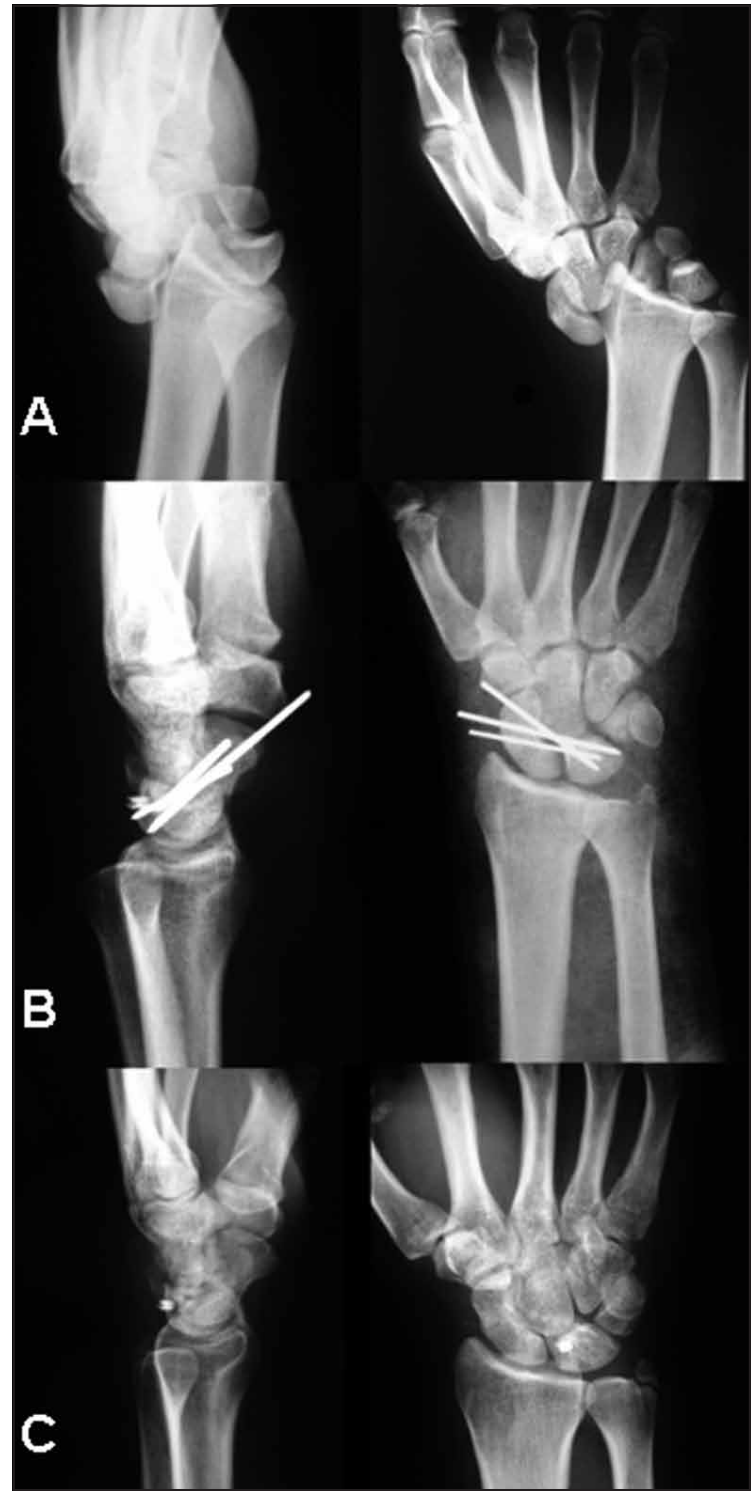

Figura 3. Imágenes radiográficas de un caso. A. Iníciales. B. postoperatorias. C. A los 6 meses tras la intervención.

sólo 21 fueron útiles para la valoración de los resultados clínicos finales, por falta de datos en 12 casos.

Los parámetros registrados fueron los siguientes: demográficos básicos, mecanismo de lesión, tiempo transcurrido entre la lesión y el diagnóstico, tipo de lesión según la clasificación de Cooney $^{8}$ (Tabla I), tiempo transcurrido entra la lesión y la instauración del tratamiento, tipo de tratamiento, si se realizó reparación ligamentosa o no, tiempo de inmovilización y resultados clínicos de acuerdo con la escala de Green y O'Brien modificada por Herzberg et al. ${ }^{7}$, esta 


\section{TABLA I - Distribución de LAS Lesiones SEgún LA CLASIFICACIÓn DE COONEY. N = NÚMERO} DE PACIENTES, \% = PORCENTAJE QUE REPRESENTA N EN LA MUESTRA

\begin{tabular}{|c|c|c|c|}
\hline & $\begin{array}{c}\text { Descripción } \\
\text { (Clasificación de Cooney) }\end{array}$ & $\mathbf{n}$ & $\%$ \\
\hline Luxac & ones perilunares $(\mathrm{I})$ & 9 & 28 \\
\hline & Transestilo (II-1) & 4 & 12 \\
\hline$\stackrel{\frac{\pi}{3}}{\frac{\pi}{2}} . \frac{0}{2}$ & Transescafo (II-2) & 12 & 36 \\
\hline ల్ల & Transescafo Transgrande (II-3) & 1 & 3 \\
\hline & II-1 o II2 + Transgrande Transpiramido (II-4) & 1 & 3 \\
\hline Luxac & ones o fracturas luxaciones axiales (III) & 1 & 3 \\
\hline Luxac & ones o fracturas luxaciones radio-carpianas (IV) & 0 & 0 \\
\hline Luxac & ón del semilunar (V) & 0 & 0 \\
\hline Trans & stilo Transescafo (No clasificables) & 5 & 15 \\
\hline Total & & 33 & 100 \\
\hline
\end{tabular}

última variable únicamente se recogió en 21 casos (Tabla II).

\section{RESULTADOS}

En esta revisión se identificó 1 luxación perilunar del carpo por cada 3.948 visitas $(0.025 \%)$. Siendo todos varones, la edad media fue de 34.7 años $(r=19-56)$. El mecanismo de lesión en 14 de ellos $(43 \%)$ fue la precipitación desde una altura mayor de 2 metros, seguido por los accidentes de tráfico en 10 casos $(30 \%)$ siendo las caídas casuales o el aplastamiento en los 9 casos (27\%) restantes. En 17 casos (52\%) se presentaron otras lesiones asociadas.

La extremidad izquierda estuvo comprometida en 18 pacientes $(55 \%)$ y no hubo casos de lesión bilateral. Seis pacientes $(18 \%)$ sufrieron lesiones abiertas. El diagnóstico se realizó el día de la lesión en 29 casos (88\%), y en los 4 (12\%) restantes la lesión paso inadvertida inicialmente diagnosticándose días después, como máximo 10 días tras el percance.

La clasificación de las lesiones encontrada puede observarse en la tabla I. Las lesiones no clasificables fueron todas fracturas-luxaciones transestilo-escafo-perilunares.

En 30 pacientes (90\%) el tratamiento se realizó en los primeros ocho días después del diagnóstico. Se sometieron a tratamiento conservador 5 pacientes $(15 \%)$ y quirúrgico a los otros $28(85 \%)$.

En los 28 casos tratados de manera quirúrgica se realizó reducción cerrada y fijación percutánea a $18(61 \%)$, mientras que en los 10 restantes (39\%) se practicó reducción abierta y fijación interna abordando la lesión por vía dorsal en 6, por vía volar en 3 y por ambas vías en 1 . De todos los pacientes en 6 se realizó reparación ligamentosa.

En el postoperatorio se mantuvo la inmovilización una media de 78 días (rango 40-125) y se realizó un seguimiento medio de 14 meses $(r=2-48)$ observando durante ese periodo las siguientes alteraciones en el curso clínico: Un paciente con una lesión Conney tipo I que tardo en diagnosticarse 8 días y se trató de manera abierta desarrolló un síndrome de dolor regional complejo. Otro paciente con una lesión Cooney tipo II-2 que se diagnosticó 10 días después de la lesión y se trató de manera percutánea, evolu- 
Tabla II - Distribución de los pacientes según los Resultados en la escala de Green Y O'Brian MOdificada POR Herzberg et AL. ${ }^{7} \mathrm{~N}=$ NÚMERO de PACIENTES, $\%=$ PORCENTAJE QUE REPRESENTA N EN LA MUESTRA

\begin{tabular}{|c|c|c|c|}
\hline \multicolumn{4}{|c|}{$\begin{array}{l}\text { RESULTADOS SEGÚN LA ESCALA DE GREEN Y O'BRIEN MODIFICADA POR } \\
\text { HERZBERG ET AL7 }\end{array}$} \\
\hline Aspecto & Valoración (puntos) & $\mathbf{n}$ & $\%$ \\
\hline \multirow{4}{*}{ Dolor (25 pts) } & Ninguno (25) & 4 & 19 \\
\hline & Leve y ocasional ( 20) & 6 & 28 \\
\hline & Moderado no en reposo (10) & 8 & 38 \\
\hline & Intenso o constante (0) & 3 & 14 \\
\hline \multirow{5}{*}{ Movilidad (25 pts) } & $>140^{\circ}(25)$ & 3 & 14 \\
\hline & $100^{\circ}-140^{\circ}(20)$ & 4 & 19 \\
\hline & $70^{\circ}-99^{\circ}(15)$ & 7 & 33 \\
\hline & $40^{\circ}-69^{\circ}(10)$ & 4 & 19 \\
\hline & $<40^{\circ}(0)$ & 3 & 14 \\
\hline \multirow{3}{*}{ Fuerza de prensión (25 pts) } & Normal (25) & 6 & 28 \\
\hline & $50 \%-90 \%(15)$ & 7 & 33 \\
\hline & $<50 \%(0)$ & 8 & 38 \\
\hline \multirow{3}{*}{ Actividades (25 pts) } & Sin restricciones (25) & 4 & 19 \\
\hline & Con restricciones (15) & 12 & 57 \\
\hline & Cambio de trabajo (0) & 5 & 23 \\
\hline
\end{tabular}

ciono hacia la necrosis del semilunar. Una lesión transestilo-transescafo-perilunar tratada percutáneamente evolucionó hacia una artrosis radiocarpiana y se trató mediante artrodesis radio-carpiana. Otro paciente con una lesión transestilotransescafo-perilunar tratada percutáneamente, presentó una seudoartrosis del escafoides que se trató de manera abierta y consolidó adecuadamente. Un paciente con una lesión Conney Tipo II-2 diagnosticada 6 días después de la lesión y tratada de manera percutánea, desarrolló una consolidación en desviación y acortamiento del escafoides, sin que requiriera tratamiento. Un paciente con una lesión Conney tipo II-4 que se trató de manera conservadora desarrolló un déficit neurológico del nervio mediano.
Al valorar el resultado clínico de los 21 paciente cuyos datos consignados en la historia clínica lo permitieron observamos en 3 (14\%) pacientes un resultado clínico excelente, 5 (24\%) bueno, 7 (33\%) pobre y 6 (29\%) malo (Tabla II). Al distribuir los resultados clínicos respecto al tipo de lesión (Tabla III) se encontraron menos resultados buenos o excelentes conforme se avanzaba en la clasificación de Cooney. Si hacemos lo mismo respecto a las lesiones abiertas observamos que ninguna de ellas puntuó como bueno o excelente resultado, siendo la puntuación en las lesiones cerradas más heterogénea (Tabla IV) Cuando observamos la distribución de los resultados clínicos según el tipo de tratamiento (Tabla V), observamos como los resulta- 
TABLA III - Distribución de Los RESULtAdos SEGÚN LA CLASIFICACIÓN DE LA LESIÓN INICIAL

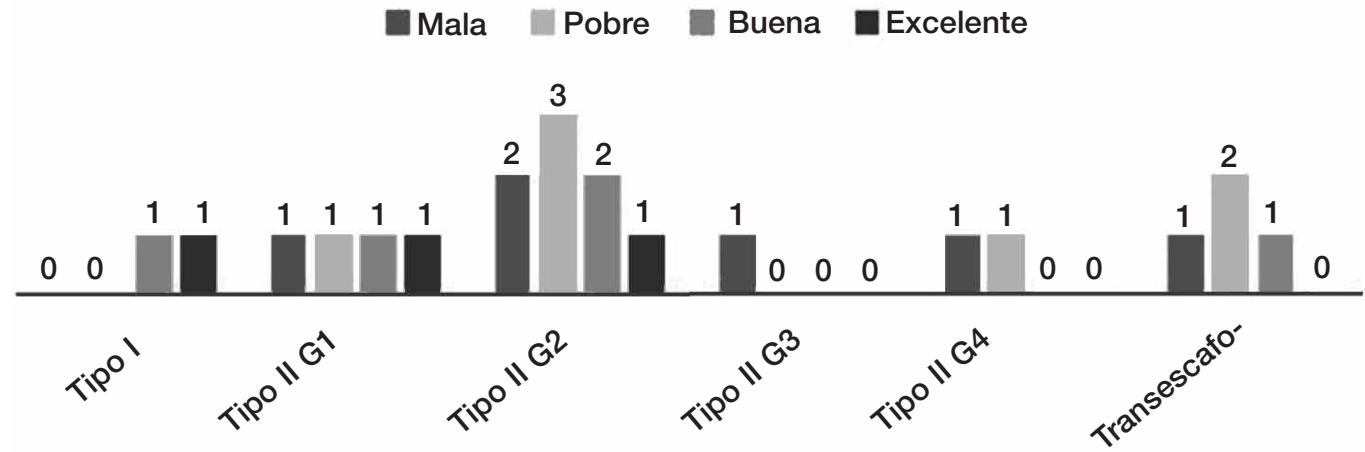

\section{TABLA IV - Distribución de los Resultados SEgún LA Lesión SEA ABIERTA o CERRADA}

Mala $\square$ Pobre Buena $\square$ Excelente

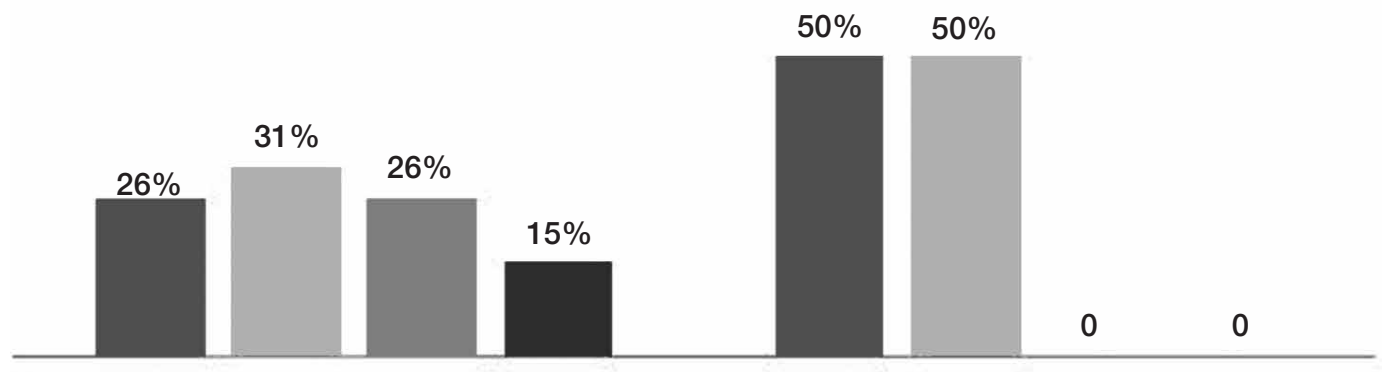

Cerrada

Abierta

\section{TABLA V - Distribución DE LOS RESULtAdos SEgúN EL TRATAMIENTO REALIZADO}

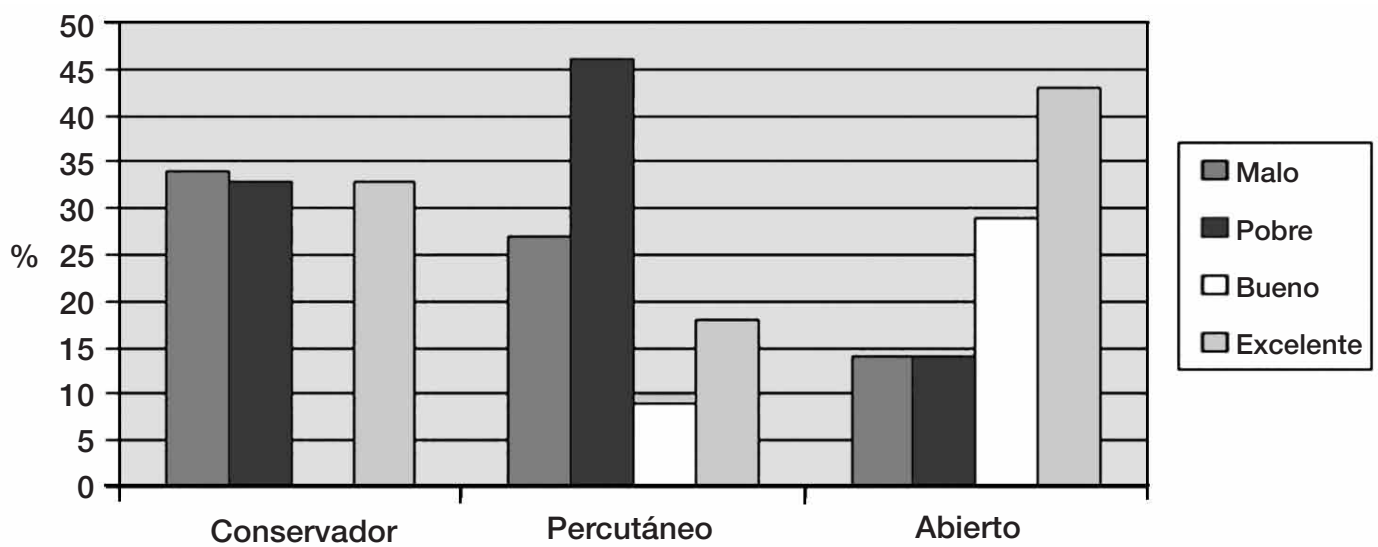

Tipo de tratamiento 
dos buenos o excelentes eran proporcionalmente más frecuentes en los casos a los que se les realizó un tratamiento mediante reducción abierta y fijación interna.

\section{DISCUSIÓN}

Este trabajo tiene múltiples limitaciones metodológicas debido a su diseño retrospectivo y a la variabilidad en la fiabilidad de los datos, ya que al tratarse de una revisión de historias clínicas, a muy largo plazo, en las que la información fue reseñada por diversos facultativos, cada uno de ellos consignó lo que consideró relevante y en 12 casos la información no fue suficiente para realizar una adecuada valoración final. Otro sesgo lo produjo el hecho de que los pacientes fueran atendidos por equipos médicos con distinta experiencia en el tratamiento quirúrgico de estas lesiones, y que el tratamiento ha sufrido cambios a lo largo de este periodo tan prolongado. Por último es una muestra pequeña que no permite realizar conclusiones estadísticas.

A pesar de todas estas limitaciones podemos señalar varios aspectos que justifican nuestro trabajo y que coinciden con otras series analizadas por distintos autores.

El hecho de que en una experiencia acumulada de 20 años -como la nuestra- se diagnosticaran 33 casos, nos indica lo poco frecuentes que son estas lesiones en nuestro medio: una cada siete meses. Esta proporción es similar a la frecuencia de la publicación de Sotereanos et al., encontrando una de estas lesiones cada 5 meses en el estudio que realizó durante 5 años ${ }^{11}$.

La totalidad de los pacientes de esta serie son de género masculino, esta prevalencia coincide con lo publicado por otros autores como Campbell ${ }^{12}$ que de los 50 casos recogidos reportaron que el $96 \%$ de los pacientes eran de género masculino.

El $12 \%$ de lesiones no diagnosticadas es una cifra elevada de retrasos diagnósticos en una patología grave y que clínicamente suele ser llamativa, sin embargo, otros autores han publicado tasas hasta del $25 \%^{8}$. Esto puede deberse a que los pacientes frecuentemente presentan lesiones de mayor gravedad en otras localizaciones lo que hace que la lesión en el carpo pase desapercibi- da, o a que el diagnóstico de la lesión se hace de manera incompleta diagnosticando únicamente la lesión ósea, recibiendo el paciente un tratamiento incompleto.

Los pacientes que fueron sometidos a reducción abierta y fijación interna mostraron mejores resultados, respecto a los que recibieron tratamiento percutáneo o conservador. Otros autores ya habían señalado los mejores resultados obtenidos mediante este tipo de tratamiento ${ }^{13}$, incluso en la importancia del abordaje por doble vía $^{12-14}$, volar y dorsal, del que en nuestra serie solo tenemos 1 caso. Sin embargo, los resultados en general son poco satisfactorios según la bibliografía consultada ${ }^{15,16}$.

Algunos autores ${ }^{17}$ han utilizado fijadores externos como tratamiento para este tipo de lesiones sin éxito, pudiendo recomendar su uso como distractor durante la cirugía.

\section{CONCLUSIONES}

Aunque nuestro estudio presenta sesgos y estadísticamente es débil, los datos recogidos reflejan que estas lesiones son poco frecuentes y en muchas ocasiones pasan desapercibidas inicialmente. Los resultados clínicos no son buenos y dependen del tipo de tratamiento, siendo mejores cuando se realiza una reducción abierta y fijación interna. Debería estandarizarse el diagnóstico y tratamiento en los servicios de urgencias con el fin de mejorar los resultados. Ya que se trata de una lesión muy grave cuyo retraso en el diagnóstico o déficit en el tratamiento comporta secuelas importantes para los pacientes.

A la vista de nuestra experiencia destacamos la utilidad de la valoración radiológica dinámica en tracción bajo anestesia local para el diagnóstico en urgencias, procediendo en el mismo acto a reducción cerrada de la lesión y, en un segundo tiempo, realizar una reparación quirúrgica abierta de forma programada por un equipo experimentado. Si es imposible la reducción cerrada debería realizarse la reducción abierta en la mayor brevedad posible.

Hay que destacar que faltan trabajos mejor diseñados para relacionar el pronóstico con el tipo de lesión o el tratamiento realizado. 


\section{BIBLIOGRAFÍA}

1. Linscheid RL. Kinematic considerations of the wrist. Clin Orthop, 1986; 202: 27-39.

2. Mayfield JK, Johnson RP, Kilcoyne RK. Carpal dislocations: Pathomechanics and progressive perilunar instability. J Hand Surg Am, 1980; :5: 226-41.

3. Mayfield JK. Patterns of injury to carpal ligaments. Aspectrum. Clin Orthop, 1984; 187: 36-42.

4. Bellinghausen HW, Gilula LA, Young LV, et al. Post-traumatic palmar carpal subluxation. Report of two cases. J Bone Joint Surg Am, 1983; 65: 998-1006.

5. Abe Y, Doi K, Hattori Y. The clinical significance of the scaphoid cortical ring sign: a study of normal wrist X-rays. J Hand Surg Eur, 2008; 33: 126-9.

6. Martín M. Luxaciones y fracturas-luxaciones perilunares del carpo. Rev Ortop Traumatol, 2007; 51 Supl 1: 124-33.

7. Herzberg G, Comtet JJ, Linscheid RL, et al. Perilunate dislocations and fracture-disloca- tions: A multicenter study. J Hand Surg Am, 1993; 18: 768-79.

8. Cooney WP. Difficult wrist fractures. Perilunate fractures-dislocations of the wrist. Clin Orthop, 1987; 214: 757-64.

9. Gaebles Ch. Fractures and dislocations of the carpus. En: Bucholz R, Heckman J, CourtBrown C (Ed). Rockwood and Green's Fractures in Adults. 6 ed. Philadelphia: Lippincott Williams \& Wilkins, 2006: 857908.

10. Garcia-Elias M, Irisarri C, Henriquez A, Abanco J, et al. Perilunar dislocation of the carpus. A diagnosis still often missed. Ann Chir Main, 1986; 5: 281-7.

11. Sotereanos DG, Mitsionis GJ, Giannakopoulos PN, et al. Perilunate dislocation and fracture dislocation: a critical analysis of the volar-dorsal approach. J Hand Surg Am, 1997; 22: 49-56.

12. Campbell RD Jr, Lance EM, Yeoh CB. Lunate and perilunate dislocations, J Bone Joint Surg $\mathrm{Br}, 1964$; 46: 55-72.

13. Weil W, Slade J, Trumble T. Open and arthroscopic treatment of perilunate injuries. Clin Orthop, 2006; 445: 120-32.

14. Trumble T, Verheyden J. Treatment of isolated perilunate and lunate dislocation with combined dorsal and volar approach and intraosseous cerclaje wire. $J$ Hand Surg Am,. 2004; 29: 412-7.

15. Hildebrand KA, Ross DC, Patterson SD, et al. Dorsal perilunate dislocations and fracture-dislocations: questionnaire, clinical, and radiographic evaluation. J Hand Surg Am, 2000; 25: 1069-79.

16. Herzberg G, Forissier D. Acute dorsal trans-scaphoid perilunate fracture-dislocations: médium-term results. J Hand Surg $\mathrm{Br}, 2002$; 27: 498-502.

17. Fernandez DL, Mader K, The treatment of complex carpal dislocations by external fixation. Injury, 2000; 31: 92-101. 